\title{
Análisis y diseño de un sistema informático para administrar el plan de trabajo del profesor de tiempo completo
}

\author{
Analysis and design of a computer system to manage the full-time teacher's work \\ plan
}

PAREDES-XOCHIHUA, Maria Petra†*, MORALES-ZAMORA, Vianney y LÓPEZ-MUÑOZ, Jesús

Instituto Tecnológico Superior de San Martín Texmelucan, Camino a Barranca de Pesos S/N San Lucas Atoyatenco San Martín Texmelucan, Puebla. C.P. 74120

ID $1^{\text {er }}$ Autor: Maria Petra, Paredes-Xochihua / ORC ID: 0000-0003-1753-2313, Researcher ID Thomson: S-6991-2018, CVU CONACYT ID: 298117

ID $1^{\text {er }}$ Coautor: Vianney, Morales-Zamora / ORC ID: 0000-0002-1181-825X, Researcher ID Thomson: S-6627-2018, CVU CONACYT ID: 308547

ID $2^{\text {do }}$ Coautor: Jesús, López-Muñoz / ORC ID: 0000-0002-3414-0891, Researcher ID Thomson: S-6861-2018, CVU CONACYT ID: 947242

DOI: $10.35429 / J T A E .2019 .10 .3 .7 .12$

Recibido: 30 de Agosto, 2019; Aceptado 20 Diciembre, 2019

Resumen

En el Instituto Tecnológico Superior de San Martín Texmelucan el Departamento de Investigación se encarga de realizar el seguimiento de la elaboración de Proyectos de Investigación y/o Desarrollo Tecnológico, los cuales en su mayoría son realizados por Profesores de Tiempo Completo (PTC). Actualmente se solicita un Plan de Trabajo semestral a los PTC's responsables de proyectos que es llenado en un formato realizado en un editor de textos, en el cual se indican cada una de las actividades a desarrollar para el proyecto, así como los productos entregables correspondientes, entre otros datos, este es entregado de manera física al responsable. En ocasiones es extraviado por error humano, por lo tanto, no se tiene la evidencia para poder dar el seguimiento en tiempo y forma. Por lo anterior, se está elaborando un sistema que permita administrar estas y otras actividades, siguiendo la Metodología Ágil de desarrollo de Software Programación Extrema para el análisis y diseño del sistema informático, que incluye las fases: Exploración, Planeación, Iteraciones, Producción y Mantenimiento. El uso del sistema permitirá tener en tiempo y forma los proyectos y así incrementar los indicadores institucionales proyectos de investigación, desarrollo tecnológico e innovación y estudiantes participantes en proyectos de investigación.

Sistema Informático, Plan de Trabajo, Profesor de Tiempo Completo

\begin{abstract}
At the Higher Technology Institute of San Martín Texmelucan the Research Department is responsible for monitoring the development of Research and/or Technological Development Projects, which are mostly performed by Full-Time Teachers. A six-monthly Work Plan is currently requested from project managers that is filled in a format made in a text editor, which indicates each of the activities to be developed for the Project, as well as the corresponding deliverable products, among other data, it is physically delivered to the controller. Sometimes it is misplaced by human error, therefore, you do not have the evidence to be able to follow up in time and form. Therefore, a system is being developed to manage these and other activities, following the Agile Methodology of Extreme Programming software development for the analysis and design of the computer system, which includes the phases: Exploration, Planning, Iterations, Production and Maintenance. The use of the system will allow to have in time and form the projects and thus increase the institutional indicators research projects, development and innovation and students involved in research projects.
\end{abstract}

Computer System, Work Plan, Full-Time Teacher

Citación: PAREDES-XOCHIHUA, Maria Petra, MORALES-ZAMORA, Vianney y LÓPEZ-MUÑOZ, Jesús. Análisis y diseño de un sistema informático para administrar el plan de trabajo del profesor de tiempo completo. Revista de Tecnología y Educación. 2019. 3-10: 7-12

\footnotetext{
* Correspondencia del Autor (petrypx @ hotmail.com)

$\dagger$ Investigador contribuyendo como primer autor.
} 


\section{Introducción}

PRODEP (2019), es el Programa para el Desarrollo Profesional (PRODEP) que busca profesionalizar a los PTC para que alcancen las capacidades de investigación-docencia, desarrollo tecnológico e innovación y con responsabilidad social, se articulen y consoliden en cuerpos académicos y con ello generen una nueva comunidad académica capaz de transformar su entorno.

Las instituciones de Educación Superior buscan que los PTC's, accedan a los apoyos que brinda PRODEP, para elevar la calidad educativa. En el Instituto Tecnológico Superior de San Martín Texmelucan ITSSMT, los PTC's participan en las convocatorias para: Apoyo a la incorporación de nuevos PTC, Apoyos para estudios de posgrado, Reconocimiento a perfil deseable y apoyo. Estas actividades son coordinadas por el responsable del Departamento de investigación, así mismo da seguimiento a los planes de trabajo semestrales que los PTC realizan para cubrir los aspectos que solicita PRODEP y participar en las convocatorias que el Tecnológico Nacional de México TecNM publica y que se podría participar en ellas. Por lo anterior, se detecta la necesidad de contar con un sistema informático en el ITSSMT que permita administrar el plan de trabajo del PTC y darles el seguimiento apropiado a estos, para mejorar los porcentajes de cumplimiento que actualmente se tienen el instituto.

El objetivo del artículo es presentar el análisis y diseño de un sistema para administrar el plan de trabajo del profesor de tiempo completo, el cual se elaborará siguiendo la metodología Extreme Programming (XP), para lo cual, se presenta el análisis y diseño correspondientes a las etapas de exploración y planeación de la metodología. Se detecta la necesidad de manejar diferentes tipos de privilegios para usuarios, y módulos que se describen en la sección de desarrollo, además de permitir acceder a la información en tiempo real, así como el acceso al sistema y a la información se realice de manera segura, asegurando la protección de los datos personales e información de los proyectos.
Con el supuesto de que sea una herramienta de apoyo para el responsable del departamento de investigación para mejorar el porcentaje de proyectos realizados en tiempo y forma, y elevar el número de productos académicos válidos para las convocatorias en las que pueda participar el PTC en beneficio de los indicadores institucionales.

\section{Antecedentes}

DOF (2014), el Tecnológico Nacional de México es creado por Decreto Presidencial el 23 de julio de 2014 como un órgano administrativo desconcentrado de la Secretaría de Educación Pública (SEP), con autonomía técnica, académica y de gestión; cuyos objetivos principales son, entre otros, los siguientes:

- Formar profesionales investigadores aptos para la aplicación y generación de conocimientos con habilidades para la solución de problemas, con pensamiento crítico, sentido ético, actitudes emprendedoras, de innovación y capacidad creativa para la incorporación para la incorporación de los avances científicos y tecnológicos que contribuyan al desarrollo nacional $\mathrm{y}$ regional.

- Desarrollar e impulsar la investigación aplicada, científica y tecnológica, que se traduzca en aportaciones concretas para mantener los planes y programas de estudio, actualizados y pertinentes, así como para mejorar la competitividad y la innovación de los sectores productivos y de servicios y elevar la calidad de vida de la sociedad.

- Fortalecer la cultura innovadora y emprendedora, así como la movilidad del personal docente y de estudiantes para incrementar su competitividad a nivel nacional e internacional.

DOF (2015), el Profesor/a de Tiempo Completo (PTC), es el personal docente que desarrolla de manera profesional una carrera de carácter académico, y que sus actividades incluyen invariablemente las funciones de docencia, tutoría de estudiantes y la generación o aplicación innovadora del conocimiento. Debe además contar con el nombramiento que acredite su dedicación de tiempo completo a la IES (Instituciones de Educación Superior) a la que pertenece. 
El Departamento Investigación es el responsable de registrar líneas de investigación, proyectos y cuerpos académicos. Otra actividad del departamento es la de realizar la Convocatoria para la captación de Proyectos de Investigación y/o Desarrollo Tecnológico del ITSSMT, en colaboración con el Departamento de Desarrollo Académico y la Subdirección Académica, también se realiza la convocatoria para la integración de estudiantes para obtener créditos complementarios.

Un plan de trabajo es una herramienta con la que se puede organizar y simplificar las actividades necesarias para concretar una acción. Con este instrumento se pueden planificar y gestionar todos los recursos necesarios para poder ejecutar dicho trabajo. También, nos ayuda a sistematizar toda la información generada, actuando de un excelente instrumento de comunicación.

El Plan de Trabajo Semestral del Profesor de Enseñanza Superior Asociado (A, B ó C) del ITSSMT es una actividad obligatoria para los PTC de cualquiera de los Programas de Estudio vigentes en el instituto. Por lo cual, cada semestre es solicitado y se entrega en el departamento de investigación, en este se deben contemplar todas las actividades que se realizaran con el objetivo de alcanzar productos académicos válidos, además de colocar información referente al docente, línea de investigación y cuerpo académico al que pertenece, entre otros datos.

\section{Desarrollo}

La metodología XP de desarrollo de software que como ya se mencionó se utilizará contempla las fases: Exploración, Planeación, Iteracciones, Producción y Mantenimiento. En el presente se describen las fases exploración y planeación.

\section{Fase de Exploración}

Se realizó una revisión y análisis de planes de trabajo de PTC, la cual fue presentada en el apartado de antecedentes. De igual forma se hizo una Exploración de las herramientas con la finalidad de conocer las características de las herramientas de software y hardware, explorar si cubren los requisitos necesarios para el desarrollo del sistema. Se debe identificar el tipo de aplicación o servicio web que se creara.
Como resultado de la exploración se identificaron los requerimientos de software que se emplearan, para el desarrollo del sistema deben ser de licencia libre con la finalidad de no generar gastos en el pago de licencias. Se utilizará XAMPP para la base de datos, la cual es una distribución de Apache completamente gratuita y fácil de instalar, que contiene MariaDB y PHP.

El editor Sublime Text se manejará como maqueteador para el desarrollo web. Material Design es un lenguaje de diseño que combina principios clásicos del diseño exitoso, así como la innovación y tecnología. El objetivo de Google en poner esta herramienta disponible para los desarrolladores consiste en desarrollar un sistema de diseño que permita una experiencia de usuario unificada a través de todos sus productos en cualquier plataforma.

Se utilizó balsamiq para realizar el diseño de las pantallas de baja fidelidad del sistema, que ayudará al desarrollador obteniendo una vista previa de las interfaces que serán implementadas, Dia para la elaboración del modelo entidad relación de la base de datos y para la programación se utilizará: CSS, JQuery, Java Script y HTML.

\section{Fase Planeación}

En esta se configuran la prioridad para las funcionalidades del usuario. Se estima cuanto esfuerzo requieren para la programación de cada funcionalidad y se hace una programación de acuerdo a esta estimación. Se identifica el orden de las prioridades de las funcionalidades establecidas por el usuario en las entrevistas, se analiza la base de datos y normaliza de acuerdo al sistema manejador de base de datos que se emplee.

La prioridad de las funcionalidades dependerá del usuario que acceda al sistema, los tipos de usuarios serán: administrador el cual será utilizado por el responsable del departamento y los PTC. Cada uno de estos tendrá diferentes privilegios. A continuación, se muestran algunas de las pantallas de baja fidelidad que se diseñaron. 
En la figura 1, se presenta la pantalla de baja fidelidad del inicio del sistema, la cual aplica para los dos tipos de usuarios, en esta se deberá ingresar el usuario y contraseña y presionar el botón ingresar, en caso de olvidar contraseña se podrá recuperar, esta pantalla cuenta con el botón de registro de usuario que al presionar mostrara la figura 2 , en la cual se solicitan los datos de nombre, número de SIE, carrera en la que se encuentra adscrito, usuario y contraseña, entre otros datos.

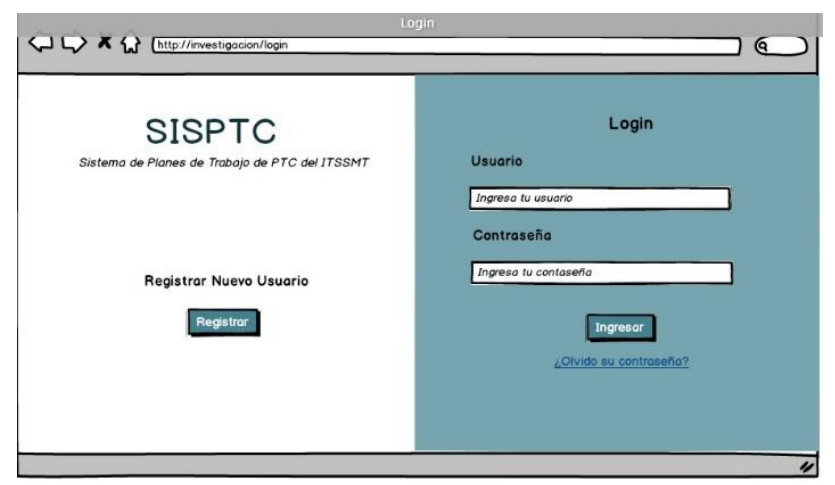

Figura 1 Pantalla inicio

Fuente: Elaboración Propia

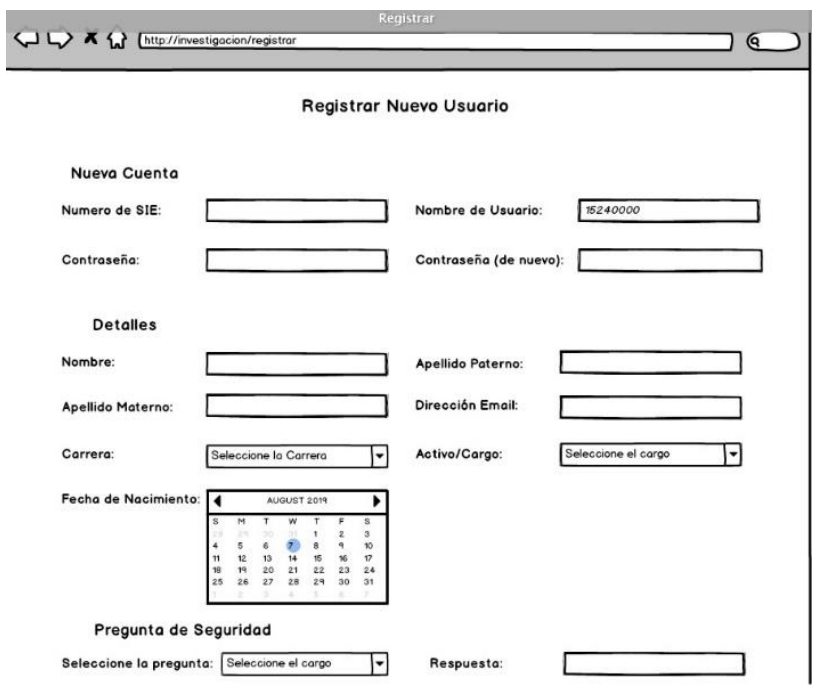

Figura 2 Pantalla Registro de usuario Fuente: Elaboración Propia

La figura 3 ilustra que datos se deberán registrar en el plan de trabajo, el cual será capturado por el usuario PTC responsable del proyecto, esta será una de las interfaces con mayor demanda de tiempo para su captura, y que el usuario administrador deberá revisar y autorizar.

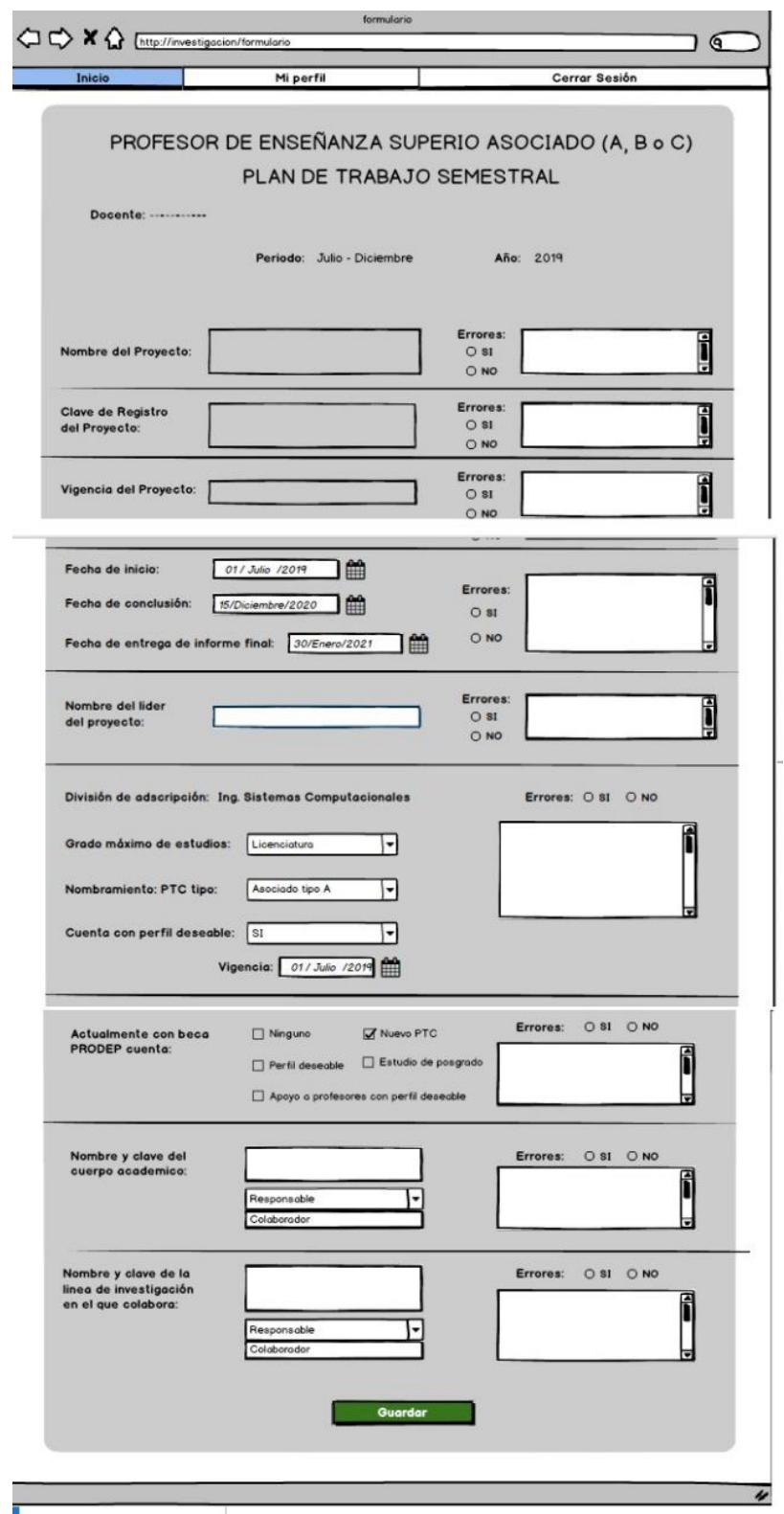

Figura 3 Pantalla registro de plan de trabajo Fuente: Elaboración Propia

El cronograma de actividades a realizar en el plan de trabajo que se observa en la figura 4 , se deberán colocar cada una de las actividades en orden de elaboración, indicando el mes en que se tendrá el producto académico, esta sección también es elaborada por el PTC e igual revisada $\mathrm{y}$ autorizada por el administrador. 


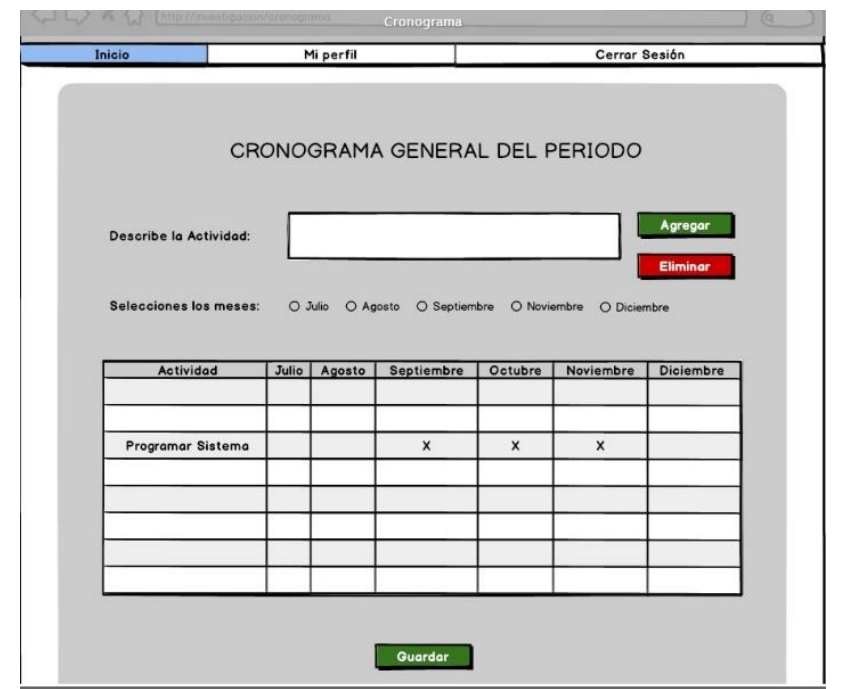

Figura 4 Pantalla de registro de cronograma Fuente: Elaboración Propia

Una vez que los PTC terminen su plan de trabajo semestral, el administrador lo deberá revisar y autorizar, por lo que este indicara si existen comentarios o lo autoriza para impresión y entrega, la figura 5 muestra la pantalla de revisión. La figura 6 , presenta el seguimiento que realizara el responsable del departamento de investigación.

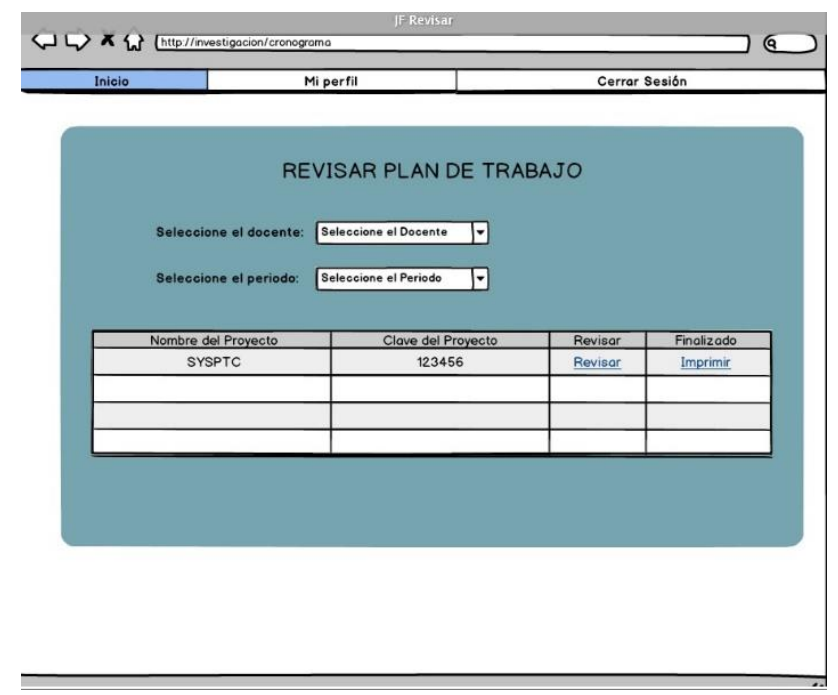

Figura 5 Pantalla de revisión de plan de trabajo Fuente: Elaboración Propia

El modelo entidad-relación de la base de datos se presenta de manera general en la figura 6. Es necesario hacer mención que se tendrá un módulo de generación de reportes e impresión de los planes de trabajo, y para tener acceso a este es necesario que los planes de trabajo deben ser autorizados por el responsable del departamento de investigación.

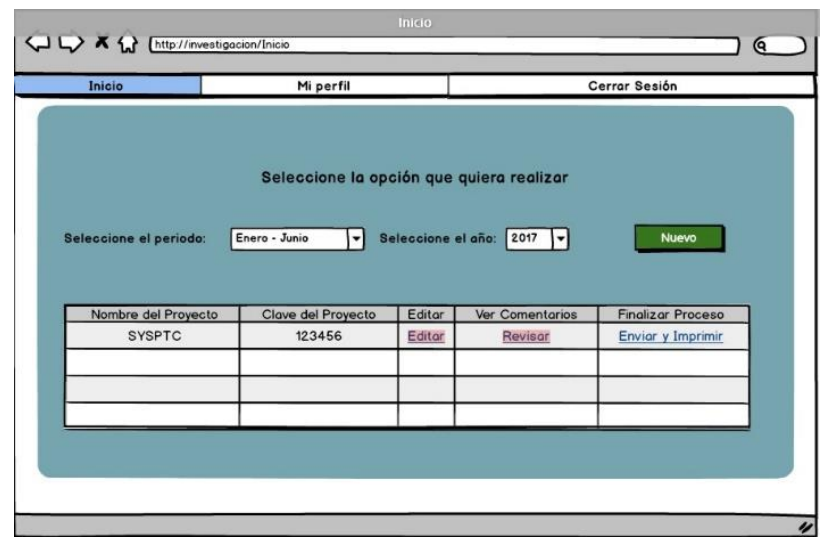

Figura 6 Pantalla de seguimiento de proyectos Fuente: Elaboración Propia

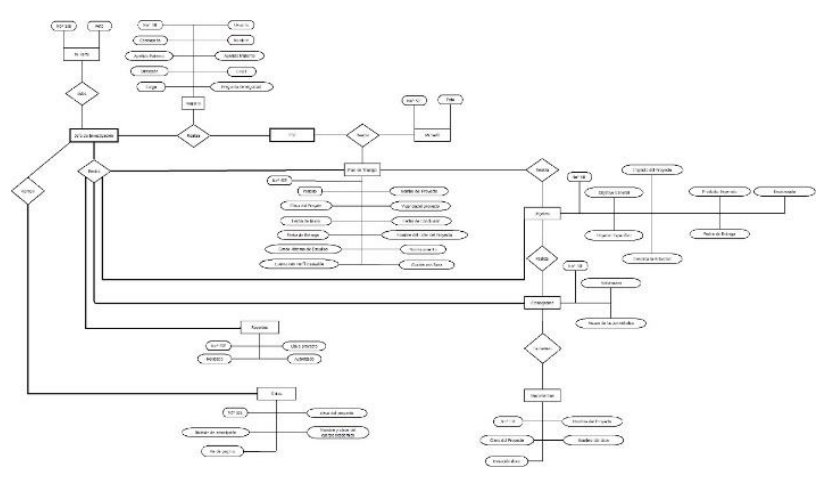

Figura 6 Modelo entidad-relación Fuente: Elaboración Propia

\section{Conclusión}

El análisis y diseño que se realizó es con la finalidad de permitir de manera automática, sencilla y segura, tener la información de los planes de trabajo de los PTC del ITSSMT. Con el propósito de mejorar los indicadores institucionales del área de investigación. Además de que el responsable del área, pueda tener un seguimiento apropiado de los proyectos en tiempo y forma. Así mismo disminuir los errores humanos y cuidar el uso del papel. Las pantallas de baja fidelidad se han realizado cuidando lo solicitado por el departamento y las variables que intervendrán en cada una de las consultas y reportes generados.

\section{Referencias}

¿Qué es CSS? (s.f.). En Libros web. Consultado el 27 de marzo de 2019. Recuperado de: http://librosweb.es/libro/css/capitulo_1.html

DOF (2014), Decreto TecNM, 2014_07_03. Consultado el 23 de Mayo de 2019. Recuperado de:

http://www.dof.gob.mx/nota_detalle.php?codig $\mathrm{o}=5353459 \&$ fecha $=23 / 07 / 2014$ 
DOF (2015), Acuerdo número 24/12/15, Reglas de Operación para el PRODEP, 2015_12_31. Consultado el 18 de Mayo de 2019. Recuperado de:

http://dof.gob.mx/nota_to_doc.php\%3Fcodnota $\% 3 \mathrm{D} 5422013$

Introduction. (s.f.) En Getting started. Consultado el 23 de marzo de 2019. Recuperado de:

https://www.muicss.com/docs/v1/gettingstarted/introduction

JavaScript basics. (s.f.). En Learn web development. Consultado el 23 de marzo de 2019. Recuperado de:

https://developer.mozilla.org/en-

US/docs/Learn/Getting_started_with_the_web/J avaScript_basics

PRODEP (2019), Programa para el Desarrollo Profesional. Consultado el 18 de Mayo de 2019. Recuperado de: https://www.dgesu.ses.sep.gob.mx/PRODEP.ht $\mathrm{m}$

Visión General de Material Design Lite. (s.f.). Consultado el 27 de marzo de 2019. Recuperado de:

https://webdesign.tutsplus.com/es/articles/anoverview-of-material-design-lite--cms-24370

What is jQuery? (s.f.). En jQuery. Consultado el 27 de marzo de 2019. Recuperado de: https://jquery.com/. 